\title{
Exciting field and quadrature-axis armature reaction in a cascade equivalent $\mathrm{A}-\mathrm{H}$-circuit of a salient-pole generator
}

\author{
Alexey Blanc \\ Department of Theoretical Foundation of Electrical Engineering, Novosibirsk State Technical University, Russia
}

\begin{tabular}{l} 
Article Info \\
\hline Article history: \\
Received May 29, 2019 \\
Revised Oct 29, 2019 \\
Accepted Nov 6, 2019 \\
\hline
\end{tabular}

\section{Keywords:}

Cascade equivalent circuits

Electric machines

Piecewise continuous Sturm-

Liouville eigenfunctions

Salient-pole electric machines

Synchronous electric generators

\begin{abstract}
Cartesian and cylindrical laminated models are well known in calculations of electric machines. In such models, general solutions of partial differential equations are transformed into four-terminal network equations, and this makes possible to synthesize cascade equivalent circuits of electric machines. In salient-pole machines, solutions of partial differential equations are formed on the base of piecewise continuous Sturm-Liouville eigenfunctions. However, in this case, cascade equivalent circuits cannot be synthesized since it needs many piecewise continuous eigenfunctions in the zone of poles and many smooth functions in the zone of the air gap for ensuring uniqueness of a solution. Meanwhile the author of this paper had offered an approximate method on the base of the single piecewise continuous Sturm-Liouville eigenfunction in the zone of poles and many smooth functions in the zone of the air gap. This method allows transforming a solution of a partial differential equation into four-terminal network equations and synthesizing cascade equivalent circuits of salient-pole machines. In this paper, electromagnetic field of a synchronous salient-pole generator is calculated with a cascade equivalent A-H-circuit. The cell that corresponds to rotor poles is synthesized on the base of a solution of Laplace's equation with the single piecewise continuous Sturm-Liouville eigenfunction.
\end{abstract}

Copyright $@ 2020$ Institute of Advanced Engineering and Science. All rights reserved.

\section{Corresponding Author:}

Alexey Blanc,

Department of Theoretical Foundation of Electrical Engineering,

Novosibirsk State Technical University,

20 Marx avenue, Novosibirsk, 630073 Russia.

Email: alblances@yandex.ru

\section{INTRODUCTION}

In the 1950-1970s, many papers appeared [1-7] where electromagnetic calculations of electric machines on the base of laminated models were considered. In this model, each layer corresponds to a certain structural zone of the electric machine (for example a rotor yoke, a stator yoke, teeth and slots, air gaps). There is integrated electromagnetic field all over the functional volume. In each layer, using simplifying assumptions, it is necessary to find a general solution of the partial differential equation, then, on the grounds of continuity conditions, to work out and solve the system of linear algebraic equations in order to determine unknown integration constants which number is twice as much layers of the model.

However, this area did not have any progress due to difficulty of calculations and unhandiness of results. At the same time, these solutions may be reduced to a system of equations of a standard four-terminal network and, on layer's boundaries, tangential components of electric and magnetic intensities can be regarded as a voltage and a current. On the grounds of continuity of tangential components of electric and magnetic intensities between layers, four-terminal networks must be connected as a cascade connection. As a result, the $E$ - $H$-cascade equivalent circuit of the laminated model will be synthesized. Various laminated models and $E-H-$, or $B-H$ - or $A-H$ - equivalent circuits of electric machines are well-known [8-20]. In addition, there are electromagnetic cascade equivalent circuits of induction heating systems [21-25]. 
In salient-pole machines, solutions of partial differential equations are formed on the base of piecewise continuous Sturm-Liouville eigenfunctions when the zone containing ferromagnetic poles and non-magnetic spaces cannot be transformed into the homogeneous domain [26-28]. However, in this case, cascade equivalent circuits cannot be synthesized since it needs many piecewise continuous eigenfunctions in the zone of poles and many smooth functions in the zone of the air gap for ensure uniqueness of a solution. Moreover, it is the quantity of smooth functions that determines accuracy of a solution in the air gap. Meanwhile the author of this paper had offered an approximate method on the base of the single piecewise continuous Sturm-Liouville eigenfunction in the zone of poles and many smooth functions in the zone of the air gap [29, 30]. This method allows to transform a solution of a partial differential equation into fourterminal network equations and to synthesize cascade equivalent circuits of a salient-pole machine [31, 32].

In this paper, a synthesis of a cascade equivalent $A$ - $H$-circuit of a synchronous salient-pole electric generator is considered and magnetic field is calculated. Let us suppose that poles are fixed on the perfect ferromagnetic rotor yoke where magnetic permeability is infinite. Magnetic permeability of poles is constant. Also, we suppose that a model has a unit length in the line of a machine axis. Because of symmetry, let us consider magnetic field in the range of half a pole pitch.

\section{AN INDUCTOR OF A SYNCHRONOUS SALIENT-POLE GENERATOR AS A STANDARD CELL OF A CASCADE EQUIVALENT $\boldsymbol{A}$ - $\boldsymbol{H}$-CIRCUIT}

Figure 1 shows the analytical model of a synchronous salient-pole generator. It should be noted that, in this model, poles and spaces between poles must be confined to coordinate surfaces of the cylindrical coordinate system. Therefore, prismatic poles must be purposively configured as sphenoid. Substituting sphenoid poles for prismatic, it is necessary to keep a pole volume invariable to ensure equivalence.

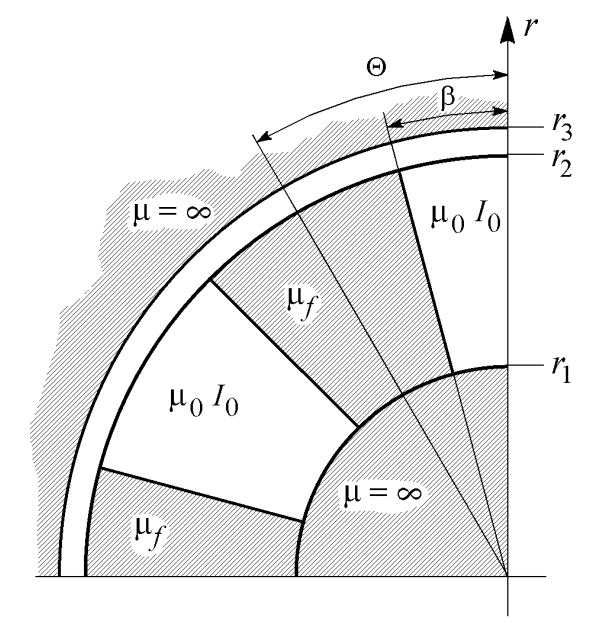

Figure 1. The analytical model of a synchronous salient-pole generator

The exciting field is generated with the current $I_{0}$ that is located in spaces between poles. Let us substitute an equivalent current sheet (that will be located on the rotor yoke between poles) for the exciting current. Fourier's expansion turns the current sheet into

$$
H_{0}(\alpha)=\sum_{k=1,3,5}^{\infty} \frac{I_{0} \sin k p \beta}{r_{1} k p \Theta \beta} \cos k p \alpha
$$

where $2 p$ is the number of poles.

In the whole zone that corresponds to alternate poles and spaces between poles, magnetic permeability may be described by the piecewise continuous function (in the range of half a pole pitch)

$$
\mu(\alpha)= \begin{cases}\mu_{0}, & 0<\alpha<\beta, \\ \mu_{f}, & \beta<\alpha<\Theta,\end{cases}
$$


and the $z$-component of a vector magnetic potential satisfies Laplace's equation.

Let the solution of Laplace's equation has the single piecewise continuous Sturm-Liouville. In case of the exciting field, a vector magnetic potential is determined as

$$
A(r, \alpha)=\left[C_{1} r^{n}+C_{2} r^{-n}\right]\left\{\begin{array}{l}
\cos n \alpha, \quad 0<\alpha<\beta \\
K \sin n(\Theta-\alpha), \beta<\alpha<\Theta
\end{array}\right.
$$

where $n$ is the first positive root of the transcendental equation

$$
\begin{aligned}
& \sin n \beta \sin n(\Theta-\beta)-\frac{\mu_{0}}{\mu_{f}} \cos n \beta \cos n(\Theta-\beta)=0 \\
& K=\frac{\cos n \beta}{\sin n(\Theta-\beta)}
\end{aligned}
$$

A tangential (to the air gap) component of a magnetic intensity vector is

$$
H_{\alpha}(r, \alpha)=-\frac{1}{\mu(\alpha)} \frac{\partial A}{\partial r}=-\frac{n}{\mu_{0} r}\left[C_{1} r^{n}-C_{2} r^{-n}\right]\left\{\begin{array}{l}
\cos n \alpha, \quad 0<\alpha<\beta \\
\frac{\mu_{0}}{\mu_{f}} K \sin n(\Theta-\alpha)
\end{array}\right.
$$

Let us expand expressions (3) and (6) to Fourier's series:

$$
\begin{aligned}
& A(r, \alpha)=\left[C_{1} r^{n}+C_{2} r^{-n}\right] \sum_{k=1,3,5}^{\infty} Q_{k} \cos k p \alpha \\
& H_{\alpha}(r, \alpha)=-\frac{n}{\mu_{0} r}\left[C_{1} r^{n}-C_{2} r^{-n}\right] \sum_{k=1,3,5}^{\infty} \Psi_{k} \cos k p \alpha
\end{aligned}
$$

where

$$
\begin{aligned}
& Q_{k}=\frac{2}{\Theta}\left[\int_{0}^{\beta} \cos n \alpha \cos k p \alpha d \alpha+K \int_{\beta}^{\Theta} \sin n(\Theta-\alpha) \cos k p \alpha d \alpha\right] \\
& \Psi_{k}=\frac{2}{\Theta}\left[\int_{0}^{\beta} \cos n \alpha \cos k p \alpha d \alpha+\frac{\mu_{0}}{\mu_{f}} K \int_{\beta}^{\Theta} \sin n(\Theta-\alpha) \cos k p \alpha d \alpha\right]
\end{aligned}
$$

When a rotor rotates (and the angular frequency is $\omega$ ), an observer sees traveling waves of functions (7) and (8), in which the $k$-th harmonic, in the complex plane, takes the form

$$
\begin{aligned}
& \dot{A}_{k}=j\left[C_{1} r^{n}+C_{2} r^{-n}\right] Q_{k} \\
& \dot{H}_{\alpha k}=-j \frac{n}{\mu_{0} r}\left[C_{1} r^{n}-C_{2} r^{-n}\right] \Psi_{k}
\end{aligned}
$$

Let the first harmonics of a vector potential and a magnetic intensity vector are known at boundaries of the piecewise zone named "poles and air spaces". If $r=r_{1}$ as shown in Figure 1 then

$$
\dot{A}_{1}=j\left[C_{1} r_{1}^{n}+C_{2} r_{1}^{-n}\right] Q_{1}
$$




$$
\dot{H}_{0} r_{1}+\dot{H}_{1} r_{1}=-j \frac{n}{\mu_{0}}\left[C_{1} r_{1}^{n}-C_{2} r_{1}^{-n}\right] \Psi_{1}
$$

where the term $\dot{H}_{0} r_{1}$ is determined as the first harmonic of the expansion (1):

$$
\dot{H}_{0} r_{1}=j \frac{\dot{I}_{0} \sin p \beta}{p \Theta \beta}
$$

If $r=r_{2}$ then

$$
\begin{aligned}
& \dot{A}_{2}=j\left[C_{1} r_{2}^{n}+C_{2} r_{2}^{-n}\right] Q_{1} \\
& \dot{H}_{2} r_{2}=-j \frac{n}{\mu_{0}}\left[C_{1} r_{2}^{n}-C_{2} r_{2}^{-n}\right] \Psi_{1}
\end{aligned}
$$

Let the equivalent $A$ - $H$-circuit corresponds to the piecewise zone named "poles and air spaces" as shown in Figure 2. In this equivalent $A$ - $H$-circuit, a vector potential is regarded as a voltage, and a tangential component of a magnetic intensity vector multiplied by a radius is regarded as a current. The ideal current source $\dot{H}_{0} r_{1}$ corresponds to the first harmonic of the current sheet (1).

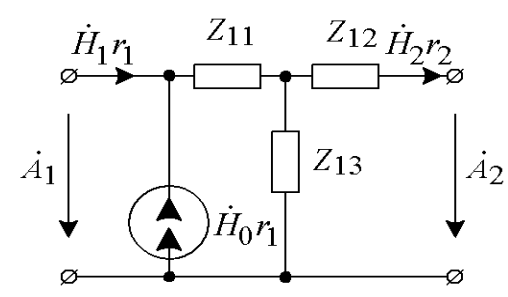

Figure 2. The equivalent $A$ - $H$-circuit of the zone named "poles and air spaces"

In the $A$ - $H$-circuit, impedances $Z_{11}, Z_{12}$ and $Z_{13}$ must not to vary when operation conditions vary. Moreover, various values of $C_{1}$ and $C_{2}$ (see expressions (13), (14), (16), (17)) correspond to various operation conditions. Analyzing expressions (13), (14), (16) and (17) in no-load conditions and short-circuit conditions, we can determine impedances as

$$
\begin{aligned}
& Z_{11}=Z_{12}=\frac{\mu_{0} Q_{1}}{n \Psi_{1}} \cdot \frac{\left(\frac{r_{2}}{r_{1}}\right)^{n}+\left(\frac{r_{1}}{r_{2}}\right)^{n}-2}{\left(\frac{r_{2}}{r_{1}}\right)^{n}-\left(\frac{r_{1}}{r_{2}}\right)^{n}} \\
& Z_{13}=\frac{\mu_{0} Q_{1}}{n \Psi_{1}} \cdot \frac{2}{\left(\frac{r_{2}}{r_{1}}\right)^{n}-\left(\frac{r_{1}}{r_{2}}\right)^{n}}
\end{aligned}
$$

If we have to calculate the quadrature-axis armature reaction then a vector magnetic potential, in the zone named "poles and spaces between poles", must be determined as

$$
A(r, \alpha)=\left[C_{1} r^{n}+C_{2} r^{-n}\right]\left\{\begin{array}{l}
\sin n \alpha, \quad 0<\alpha<\beta \\
K \cos n(\Theta-\alpha), \beta<\alpha<\Theta
\end{array}\right.
$$


where the parameter $n$ is the first (or second) positive root of a transcendental equation

$$
\begin{aligned}
& \frac{\mu_{0}}{\mu_{f}} \sin n \beta \sin n(\Theta-\beta)-\cos n \beta \cos n(\Theta-\beta)=0 \\
& K=\frac{\sin n \beta}{\cos n(\Theta-\beta)}
\end{aligned}
$$

Moreover, in case of the quadrature-axis armature reaction, the ideal current source $\dot{H}_{0} r_{1}$ must be removed from the $A$ - $H$-circuit, and

$$
\begin{aligned}
& Q_{1}=\frac{2}{\Theta}\left[\int_{0}^{\beta} \sin n \alpha \sin p \alpha d \alpha+K \int_{\beta}^{\Theta} \cos n(\Theta-\alpha) \sin p \alpha d \alpha\right] \\
& \Psi_{1}=\frac{2}{\Theta}\left[\int_{0}^{\beta} \sin n \alpha \sin p \alpha d \alpha+\frac{\mu_{0}}{\mu_{f}} K \int_{\beta}^{\Theta} \cos n(\Theta-\alpha) \sin p \alpha d \alpha\right]
\end{aligned}
$$

\section{THE CASCADE EQUIVALENT $A$-H-CIRCUIT OF THE SALIENT-POLE GENERATOR}

Figure 3 shows the cascade equivalent A-H-circuit of the synchronous salient-pole generator. The first cell $\left(Z_{11}, Z_{12}, Z_{13}\right)$ corresponds to rotor poles. The second cell $\left(Z_{21}, Z_{22}, Z_{23}\right)$ corresponds to the air gap. Constants of this cell are given in [15]. For the first field harmonic.

$$
\begin{aligned}
& Z_{21}=Z_{22}=\frac{\mu_{0}}{p} \frac{\left(\frac{r_{3}}{r_{2}}\right)^{p}+\left(\frac{r_{2}}{r_{3}}\right)^{p}-2}{\left(\frac{r_{3}}{r_{2}}\right)^{p}-\left(\frac{r_{2}}{r_{3}}\right)^{p}} \\
& Z_{23}=\frac{\mu_{0}}{p} \frac{2}{\left(\frac{r_{3}}{r_{2}}\right)^{p}-\left(\frac{r_{2}}{r_{3}}\right)^{p}}
\end{aligned}
$$

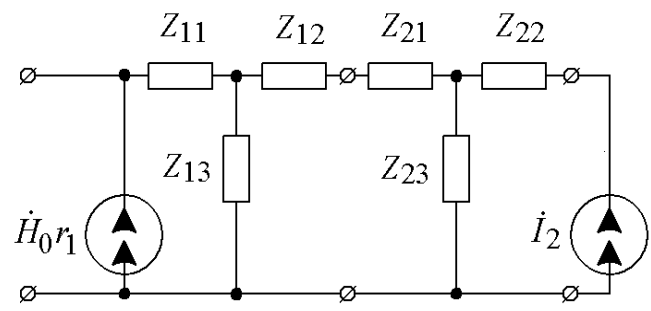

Figure 3. The cascade equivalent $A-H$-circuit of the synchronous salient-pole generator

To take into account the air gap irregularity, it is reasonable to multiply the air gap by

$$
\frac{k_{\delta}}{\alpha_{\delta}}
$$

where $k_{\delta}$ is Carter's factor, and $\alpha_{\delta}$ is the pole overlap factor. 
If magnetic permeability of stator teeth is infinite then the zone of stator teeth may be removed from the cascade equivalent $A$ - $H$-circuit and the ideal current source $\dot{I}_{2}$ (that corresponds to the armature reaction) should be connected to the output of the second cell. In compliance with Ampere's circuital low, this current source is determined as

$$
\dot{I}_{2}=\dot{\delta} h \frac{b_{1}+b_{2}}{4 \pi} z_{2}
$$

where $b_{1}$ is the slot width on the air gap; $b_{2}$ is the slot width on the stator yoke; $h$ is the tooth height; $z_{2}$ is the number of stator teeth; $\dot{\delta}$ is the current density in stator slots (the first harmonic amplitude).

After calculating vector potentials and tangent components of a magnetic intensity vector in the cascade equivalent $A$ - $H$-circuit, an electric intensity vector and a normal component of a magnetic induction vector on the rotor and on the stator are determined (for the first field harmonic) as

$$
\begin{aligned}
& \dot{E}=-j p \omega \dot{A} \\
& \dot{B}_{n}=j \frac{p}{r} \dot{A}
\end{aligned}
$$

\section{TEST RESULTS}

To verify the cascade equivalent $A$ - $H$-circuit, tests were carried out: calculations by means of the cascade equivalent $A$ - $H$-circuit and numerical simulations (ELCUT 5.1). Calculation data are given. The number of poles is 8 ; the rotor diameter is $632 \mathrm{~mm}$; the pole height is $83.4 \mathrm{~mm}$; the pole relative permeability is $500 ; \Theta=2 \beta$; the air gap is $4 \mathrm{~mm}$; the number of stator teeth is 96 ; the tooth height is $53 \mathrm{~mm}$; the slot width (on the air gap) is $11.11 \mathrm{~mm}$; the slot width (on a stator yoke) is $14.57 \mathrm{~mm}$; Carter's factor is 1.22 ; the pole overlap factor is 0.73 ; the exciting current is $4582.5 \mathrm{~A}$; the current density in stator slots is $2 \mathrm{~A} / \mathrm{mm}^{2}$ (the first harmonic amplitude, the quadrature-axis armature reaction).

Figure 4 shows the normal component of a magnetic induction vector on the stator's surface (the exciting field and the quadrature-axis armature reaction, the first field harmonic). A solid line corresponds to the cascade equivalent circuit and points correspond to the numerical simulation. Calculations of the cascade equivalent $A-H$-circuit and the numerical simulation give identical results. That indicates correctness of modeling.

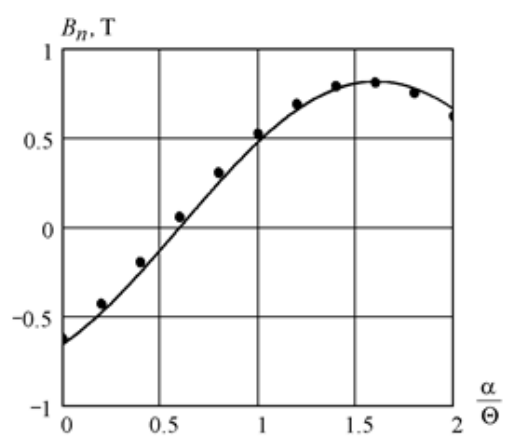

Figure 4. The normal component of a magnetic induction vector on the stator's surface (the first fieldn harmonic): a solid line corresponds to the cascade equivalent $A$ - $H$-circuit; points correspond to the numerical simulation

\section{CONCLUSION}

The author offers to use a cascade equivalent $A$ - $H$-circuit of a synchronous salient-pole generator for modeling the electromagnetic field. This cascade equivalent $A-H$-circuit is synthesized by fundamentals of the electromagnetic theory and the circuit theory. In the $A$ - $H$-circuit, prismatic poles must be configured as sphenoid. Substituting sphenoid poles for prismatic, it is necessary keep a pole volume invariable. Depending 
on the field configuration (the exciting field or the quadrature-axis armature reaction), constants of the equivalent $A$ - $H$-circuit of the zone named "poles and spaces between poles" change. To take into account the air gap irregularity, it is reasonable to increase the air gap. As follows from calculations, the cascade equivalent $A$ - $H$-circuit and numerical simulations give identical results that indicate correctness of modeling.

\section{REFERENCES}

[1] E. Mishkin, "Theory of the squirrel-cage induction motor derived directly from Maxwell's field equations," Quarterly J. of Mechanics and Appl. Mathem., vol. 7, iss. 4, pp. 472-487, 1954.

[2] S. A. Nasar, "Electromagnetic theory of electrical machines," IEE Proc., vol. 111, iss. 6, pp. 1123-1131, 1964.

[3] J. Greig and E. M. Freeman, "Traveling wave problem in electrical machine," IEE Proc., vol. 114, iss. 11, pp. 1681-1683, 1967.

[4] S. Williamson, "The anisotropic layer theory of induction machines and induction devices," IMA Journal of Applied Mathematics, vol. 17, iss. 1, pp. 69-84, 1976.

[5] V. M. Kazansky and A. I. Inkin, "An electromagnetic model and elements of the induction machine's theory," Induction Micro-Machines. The Inter-University Conference on Induction Machines: Proc. (Kaunas) pp 217-229, 1969.

[6] V. M. Kazansky, et al., "The analytical study of electromagnetic field in an active volume of the n-phase asynchronous machine with a non-slot stator," Induction electric motors with an allocated active stator layer: Proc. iss. 2, pp 41-57, Novosibirsk: NETI, 1972.

[7] A. I. Inkin and V. M. Kazansky, "Equivalent transformations of active zones in rotating electric machines," Electricity, vol. 1, pp. 42-45, 1975.

[8] E. M. Freeman, "Travelling waves in induction machines: input impedance and equivalent circuits," IEE Proc., vol. 115, iss. 12, pp. 1772-1776, 1968.

[9] E. M. Freeman, "Equivalent circuits from electromagnetic theory low-frequency induction devices," IEE Proc., vol. 121, iss. 10, pp. 1117-1121, 1974.

[10] E. M. Freeman and T. G. Bland, "Equivalent circuit of concentric cylindrical conductors in an axial alternating magnetic field," IEE Proc., vol. 123, iss. 2, pp. 149-152, 1976.

[11] A. I. Inkin, "The circuit approximation of linear mediums influenced by electromagnetic field," Electricity, vol. 4, pp. 64-67, 1975.

[12] A. I. Inkin and B. V. Litvinov, "The synthesis of cascade equivalent circuits of induction electric machines on the basis of standard E-H-four-terminal networks," Electrotechnics, vol. 1, pp. 29-33, 1977.

[13] A. I. Inkin and Yu. G. Buchholz, "Foundations of the synthesizing of non-linear cascade equivalent circuits of an electric machine," Electricity, vol. 6, pp. 33-37, 1979.

[14] A. I. Inkin, "Electromagnetic fields and parameters of electric machines: tutorial," Novosibirsk: UKEA, pages 464, 2002.

[15] B. V. Litvinov and O. B. Davidenko, "Standard cells and cascade equivalent circuits of electric machines: monograph," Novosibirsk: NSTU, pages 215, 2008.

[16] B. V. Litvinov and O. B. Davidenko, "The equivalent circuit of the synchronous reactive motor subject to higher spatial harmonic of magnetomotive force," Electricity, vol. 5, pp. 48-52, 2010.

[17] B. V. Litvinov and O. B. Davidenko, "Synchronous reactive motor with reduced magnetic conductance along transverse axis," Electrotechnics, vol. 3, pp. 15-20, 2010.

[18] L. Qaseer, et al., "Combined field and circuit theories in squirrel-cage induction motors based on micro-T circuit model," Aces Journal vol. 26, no. 7, pp. 551-560, 2011.

[19] L. Qaseer, et al., "Closed-form analysis of squirrel-cage induction motors with anisotropic modeling of stator and rotor," IEEE Trans. on Energy Conversion vol. 27, no. 3, pp. 553-560, 2012.

[20] V. V. Korneev, "Design coefficients and added losses of synchronous machines with permanent magnets and fractional tooth windings: candidate's dissertation: speciality 05.09.01," Novosibirsk: NSTU, pages 144, 2018.

[21] A. I. Inkin, et al., "The calculation of transformer induction heating system," Electrotechnics, vol. 11, pp. 34-37, 2000.

[22] F. N. Sarapulov, et al., "Mathematic models of linear induction machines on the basis of equivalent circuits," 2nd edition, Yekaterinburg: UGTU-UPI, pages 431, 2005.

[23] L. Qaseer, "Analysis of double and single sided induction heating systems by layer theory approach," Journal of electromagnetic analysis and applications, vol. 2, iss. 7, pp. 403-410, 2010.

[24] L. Qaseer, "Micro-T circuit model for double and single sided induction heating systems," Applied computational electromagnetics society journal, vol. 25, iss. 8, pp. 713-721, August 2010.

[25] L. Qaseer, "Micro-T circuit model for the analysis of cylindrical induction heating systems," IEEE Trans. on energy conversion, vol. 25, iss. 4, pp. 1021-1027, Dec. 2010.

[26] A. I. Inkin, "The analytical treatment of magnetic field in the active volume of the electric machine with permanent magnets," Electricity, vol. 5, pp. 30-33, 1979.

[27] A. I. Inkin, "Analitical solution of magnetic field's equations in discrete structures of salient-pole electric machines," Electricity, vol. 8, pp. 18-21, 1979.

[28] A. I. Inkin, "The mathematical formulation of magnetic field in the volumes of salient-pole the electric machines," Electricity, vol. 2, pp. 30-35, 1997. 
[29] A. V. Blanc, "The analytical calculation of exciting field of synchronous machine on the base of the single piecewise continuous eigenfunction," Collected scientific papers of the NSTU, vol. 4(38), pp. 3-8, 2004.

[30] A. I. Inkin and A. V. Blanc, "The approximate analytical calculation of exciting field of electric machines on the base of the piecewise continuous eigenfunction," Electricity, vol. 6, pp. 52-56, 2008.

[31] A. V. Blanc, "The A-H-equivalent circuit of a synchronous electric machine with tangential permanent magnets," Proceedings of the 2018 14th international scientific-technical conference on actual problems of electronic instrument engineering (APEIE-44894), vol. 1, part 5, pp. 62-65, 2018.

[32] A. V. Blanc, "A cascade equivalent A-H-circuit of a permanent magnet excited electrical machine," Electricity, vol. 10, pp. 42-47, 2019.

\section{BIOGRAPHY OF AUTHOR}

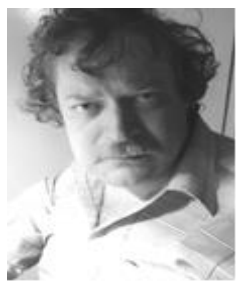

Alexey Blanc is the Candidate of Technical Science, the associate professor in the Department of the Theoretical Electrical Engineering in the Novosibirsk State Technical University. He was born in 1971 and graduated from the Novosibirsk State Technical University in 1999. In 2005, he had defended his thesis "The development of the method for calculation of magnetic field in piecewise homogeneous cylindrical zones of salient-pole the electric machines" (in Russian). He has 60 scientific publications. His scientific line relates to the engineering electrodynamics and the heattransfer theory 